\title{
Crescimento e mudança demográfica: uma aplicação do modelo de Goodwin para países da OCDE (1960-2010)
}

Growth and demographic change: An application of the Goodwin model for OECD countries (1960-2010)

\author{
Daniel Nogueira Silva(1) \\ Henrique Morrone (2) \\ (1) Universidade Federal do Sul e Sudeste do Pará \\ (2) Universidade Federal do Rio Grande do Sul
}

\begin{abstract}
The objective of this article is to evaluate the empirical adherence of the Goodwin Model (1967), when considering the inclusion in this model of a social security system characterized by a homogeneous rate of exogenously determined social security tax and adjustable individual pension levels for a determinant number of retirees. The empirical analysis is applied to a set of economies developed in the period from 1960 to 2010. Based on the data and on the econometric methodology used, the results found point to a better empirical adjustment of the Goodwin model (1967), which indicates that the Population aging can affect economic cycles through the social security system.
\end{abstract}

\section{Keywords}

Goodwin's model, populations ageing, OECD.

JEL Codes O47, J26, O15.

\section{Resumo}

O objetivo deste artigo é avaliar a aderência empirica do modelo de Goodwin (1967) quando se incorpora a esse modelo um sistema de seguridade social caracterizada por uma alíquota homogênea de imposto previdenciária exogenamente determinada e niveis de pensão individuais ajustáveis para um determinando número de aposentados. A análise empírica é aplicada para um conjunto de economias desenvolvidas e abrange o periodo de 1960 a 2010. Com base nos dados e na metodologia econométrica utilizada, os resultados encontrados apontam para um melhor ajustamento empirico do modelo de Goodwin (1967), indicando que o envelhecimento populacional pode afetar os ciclos econômicos via sistema de seguridade social.

\section{Palavras-chave}

modelo de Goodwin, envelhecimento populacional, OCDE.

Códigos JEL O47, J26, O15. 


\section{Introdução}

O trabalho de Goodwin (1967) é uma representação útil da macrodinâmica do conflito social que acompanha a distribuição de renda. O núcleo desse modelo é formalizado para apresentar a interação entre trabalhadores e capitalistas, de modo a descrever a dinâmica entre a taxa de ocupação da força de trabalho e a participação dos trabalhadores no produto. Desde a sua publicação, o modelo de Goodwin (1967) foi explorado em várias direções, incluindo o realismo de suas suposições, a sua estabilidade e possíveis generalizações. Apesar de existirem diversas limitações, a riqueza da abordagem de Goodwin pode ser observada pelos links que são construídos com diferentes autores, como Minsky (Batisdas et al., 2019; Rammelt, 2019), Kaldor (Taylor et al., 2018), Schumpeter (Flaschel, 2009).

De maneira geral, os trabalhos utilizando a teoria de Goodwin (1967) se dividem em dois grupos: de um lado estão os estudos teóricos que buscam analisar a estabilidade estrutural do modelo, e do outro, os que o testam empiricamente. Tratando da estabilidade, segundo Gandolfo (1980), o sistema de Lotka e Voterra, base para o modelo de Goodwin, não possui estabilidade estrutural. Isso ocorre porque nenhuma das variáveis que são tomadas para esse sistema podem permanecer absolutamente constantes ao longo do movimento do sistema presa-predador. Assim, uma pequena perturbação pode alterar o comportamento do sistema, o que modifica qualitativamente sua dinâmica.

Por se basear nesse sistema, o problema da estabilidade do modelo de Goodwin é recorrentemente citado na literatura (Pichardo, 2009). Sendo que uma parte importante da literatura visa estender o modelo de Goodwin para eliminar essa característica tida como indesejável (Sportelli, 1995; Veneziani, 2001; Veneziani; Mohun, 2006). Contudo, como apontado por Veneziani e Mohun (2006), há também autores que consideram a instabilidade estrutural como um elemento inerente a uma interpretação dialética e não mecanicista da teoria marxista.

Além dos trabalhos que analisam a estabilidade do modelo, muitos autores utilizaram a análise de Goodwin para testar empiricamente a interação entre o grau de pleno emprego e a participação dos salários a partir de diferentes metodologias. O primeiro trabalho a testá-lo empiricamente usando simulações matemáticas foi o de Atkinson (1969). Depois dele, diversos trabalhos utilizaram o modelo de Goodwin para entender fenô- 
menos empíricos a partir de diferentes metodologias. ${ }^{1}$ Goldstein (1999) usou a metodologia dos Vetores Autorregressivos (VAR) para entender a interação dinâmica entre desemprego e participação dos lucros na renda. Outra contribuição importante dentro da discussão empírica de Goodwin (1967) foi o trabalho de Harvie (2000). Nesse artigo, o autor analisa os ciclos econômicos de Goodwin em dez países da Organização para Cooperação e Desenvolvimento Econômico (OCDE). As principais conclusões desse trabalho trazem evidências qualitativas para o modelo de Goodwin, mas que não são confirmados quantitativamente.

Desde sua publicação, esse trabalho foi citado como uma referência para testes empíricos de modelos de ciclo de crescimento dinâmico (Molina; Medina, 2010; Tarassow, 2010). Contudo, segundo Grasselli e Maheshwari $(2017 ; 2018)$, os problemas na metodologia e na construção dos dados presentes nele geram resultados equivocados e precisam ser revistos. Ao corrigirem esses erros, os autores alcançam um ajuste melhor das estimações, o que tornam os seus trabalhos importantes referências para analisar empiricamente o modelo de Goodwin (1967) (Araujo et al., 2019).

Buscando contribuir para a literatura que trata dos aspectos empíricos, o objetivo deste artigo é avaliar o ajuste do modelo de Goodwin (1967), usando a metodologia de Harvie (2000) retificada por Grasselli e Maheshwari (2018), ao se considerar a inclusão nesse modelo de um sistema de seguridade social caracterizada por uma alíquota de imposto previdenciário homogênea e exogenamente determinada e níveis de pensão individuais, ajustáveis para um determinando número de aposentados, como apresentado no ajuste empírico do Modelo A proposto por Rada (2012). Esse último trabalho expande o modelo de Goodwin incorporando o envelhecimento da população a partir de quatro modelos baseados nos diferentes pressupostos em relação ao imposto previdenciário e à mudança tecnológica. Desses, apenas no terceiro modelo (Modelo C), o imposto de seguridade social pode levar o modelo em direção à instabilidade. Nos demais, incluindo o que é aplicado neste trabalho, o sistema é resolvido

1 Para uma revisão detalhada dos trabalhos empíricos com base no modelo de Goodwin (1967), ver Araujo et al. (2019). Nesse trabalho, além de fazerem uma revisão dos principais trabalhos empíricos, os autores também apresentam os trabalhos teóricos. Dávila-Fernández e Libânio (2016) exploram os ciclos de Goodwin e os modelos de restrição do balanço de pagamentos. Para um artigo mais recente que modela os ciclos de Goodwin empregando sistemas P-fuzzy, ver Sánchez et al. (2020). 
em um ponto fixo não trivial. ${ }^{2}$ Algumas conclusões principais do trabalho de Rada (2012) indicam que um aumento nas taxas de seguridade social reduziria a taxa de emprego, embora a distribuição primária de renda tenha se mantido inalterada. Além disso, o progresso técnico pode melhorar a sustentabilidade econômica diante de um processo de envelhecimento da população. Segundo a autora, a transição demográfica pode afetar as relações de produção nas economias capitalistas e ajudaria a explicar a dinâmica do modelo original de Goodwin.

Para avaliar o modelo de Goodwin (1967) expandido por Rada (2012), foram utilizadas duas metodologias econométricas, com base em Grasselli e Maheshwari (2018), para estimar os parâmetros do modelo: o método de Mínimos Quadrados Ordinários e a modelagem Autoregressive-Distributed Lag (ARDL). Os bancos de dados macroeconômicos utilizados no modelo foram o da Direção Geral da Comissão Europeia para Assuntos Econômicos e Financeiros - European Commission's Directorate General for Economic and Financial Affair (AMECO) - e o da Organização para Cooperação e Desenvolvimento Econômico (OCDE). Com base nesses dados e na metodologia aplicada, o modelo desenvolvido por Rada (2012) é testado para nove países: Austrália, Canadá, Dinamarca, Finlândia, França, Grécia, Itália, Reino Unido e EUA, no período de 1960 a 2010, e os resultados obtidos comparados com outros trabalhos empíricos baseados no modelo original de Goodwin, em especial Harvie (2000). As principais conclusões apontam para um bom ajustamento empírico do modelo de Goodwin (1967), em linha com alguns dos resultados alcançados por Grasselli e Maheshwari (2018), indicando que o envelhecimento populacional pode afetar os ciclos econômicos via sistema de seguridade social.

Para alcançar os objetivos do presente artigo, além desta introdução, este artigo está organizado da seguinte forma: na seção 2 é apresentado o modelo original de Goodwin do ciclo distributivo, que é a referência inicial para a análise desenvolvida neste artigo; na seção 3, com base na contribuição de Rada (2012), o modelo de Goodwin é expandido de modo a incorporar o problema da transição demográfica dentro do conflito distributivo; na seção 4, são apresentados os dados e a metodologia; na seção 5, são analisados os resultados. Finalmente, a seção 6 apresenta as considerações finais.

2 Rada (2012) aplicou o critério de estabilidade Routh-Hurwitz para testar matematicamente a estabilidade dos modelos, e o resultado dos Jacobianos da Equação aponta que "the system is likely to be stable" (Rada, 2012, p. 741). 


\section{Modelo de Goodwin}

O modelo proposto por Goodwin (1967) é essencialmente um sistema presa-predador de Lotka e Volterra que formaliza o conflito de classes descrito por Marx em $O$ capital. Contudo, antes de tratar das bases teóricas, é importante entender os fundamentos metodológicos desse modelo. O sistema proposto por Lotka e Volterra, no qual Goodwin se inspira, trata-se de um instrumento matemático para analisar a interação entre presa e predadores, comumente utilizados em estudos no campo da Biologia.

O modelo de Goodwin (1967) é uma formalização da explicação de Marx (1976) do conflito de classes sobre a apropriação do excedente em sua teoria da acumulação capitalista, com base na mesma dinâmica desenvolvida por Lotka e Volterra. Nesse caso, a participação dos salários na renda seria o predador, variável de distribuição associada aos trabalhadores, e o nível de emprego, as presas, variável associada aos capitalistas. A dinâmica central é uma relação cíclica entre a participação dos salários na renda e a taxa de emprego. Esses movimentos são geralmente associados ao curto prazo (Shaikh, 2016), o que muitas vezes dificulta uma análise empírica, visto que os dados para muitos países estão disponíveis apenas em nível anual (Veneziani; Mohun, 2006). O ponto de partida utilizado por Goodwin para entender o conflito entre trabalhadores e capitalistas é o Livro I, volume II de O capital, de Karl Marx (1983), especialmente o capítulo XXIII, em que o autor apresenta a Lei Geral da Acumulação Capitalista.

Os principais pressupostos teóricos da Lei Geral de Acumulação Capitalista de Marx (1983) foram transformados num modelo matemático por Goodwin, formalmente baseado num conjunto de hipóteses reduzidas a um par de equações diferenciais que representam a participação dos salários na renda nacional $(\omega)$ e a taxa de emprego $(\lambda)$.

O modelo assume uma economia fechada sem governo. Além dessas características, ele pode ser descrito conforme as seguintes hipóteses:

(A1) Progresso técnico é constante, tal que a produtividade do trabalho (a) cresce a uma taxa constante cresce a uma taxa constante $(x)$, e é dada por $\left(a=A_{0} e^{x t}\right)$.

(A2) Crescimento exógeno estável da força de trabalho, de modo que a oferta de trabalho $(N)$ cresça a uma taxa constante $(n)$, e é dada por $\left(N=N_{0} e^{x t}\right)$. 
Cabe destacar que tanto $A_{0}$ quanto $N_{0}$ nas hipóteses (A1) e (A2) são constantes paramétricas estritamente positivas exogenamente determinadas.

(A3) Existem apenas dois fatores de produção homogêneos e não específicos: capital e trabalho.

(A4) Todas as quantidades são reais e líquidas.

(A5) Todos os salários ( $w L$ ) são consumidos e todos os lucros $(\Pi=X-w L)$ são poupados e reinvestidos. Sendo que w é o salário, $L$ é o número de trabalhadores empregados e $X$ é o produto.

(A6) Existe uma relação capital-produto (inverso da produtividade do capital) constante $(\sigma=K / X)$, sendo $K$ o estoque de capital.

(A7) Um salário real que aumenta próximo do pleno emprego, expresso pela curva de Phillips ( $\dot{w} / w=-\eta_{1}+\eta_{2} \lambda$ ), sendo que $\eta_{1}$ é o intercepto da Curva, $\eta_{2}$ a elasticidade, e $\lambda$ a taxa de emprego.

Partindo dessas hipóteses, o modelo descreve órbitas fechadas no espaço $(w, \lambda)$ de acordo com as seguintes equações fundamentais:

$$
\begin{aligned}
& \dot{\omega}=\left[-\left(x+\eta_{1}\right)+\eta_{2} \lambda\right] \omega \\
& \dot{\lambda}=[(1 / \sigma-(x+n)-\omega / \sigma] \lambda
\end{aligned}
$$

A equação 1 refere-se à parcela salarial $(\dot{\omega})$, e a 2 , a taxa de emprego ou utilização da capacidade $(\dot{\lambda})$. Com exceção de casos excepcionais em que os valores de $\lambda$ podem ser maiores do que 1 , especialmente nas situações em que os salários e o consumo são maiores do que o produto, as duas variáveis serão quase sempre menores que 1.

$\mathrm{Na}$ equação 1 , a primeira expressão $\left.\left[\left(x+\eta_{1}\right) \omega\right)\right]$ serve para indicar os fatores que têm um impacto negativo na participação dos salários no produto. A variável $x$ representa a taxa de crescimento do progresso técnico. Nesse caso, quando o valor do parâmetro aumenta, diminui a barganha salarial dos trabalhadores, o que leva a uma redução dos salários. $\bigcirc \eta_{1}$ é o intercepto linear da curva de Philips, a qual indica que há um trade-off entre a barganha salarial e o desemprego. A segunda expressão da equação $\left(\eta_{2} \lambda \omega\right)$ mostra que o crescimento da participação salarial é proporcional à taxa de emprego e à elasticidade do salário em relação ao emprego.

$\mathrm{Na}$ equação 2, referente à taxa de emprego, a primeira expressão $[(1 / \sigma-(x+n))]$ indica a taxa de crescimento do emprego, caso a parcela salarial $(\omega)$ seja igual a zero. Sendo que $(1 / \sigma)$ é a produtividade do capital 
(suposta constante), e $(x+n)$, a taxa natural de crescimento. Para que haja crescimento do emprego, basta que a produtividade do capital seja maior do que a taxa natural de crescimento. A segunda expressão da equação 2 $[(\omega / \sigma) \lambda]$ é onde aparecem os fatores que provocam o desemprego, sendo que $\sigma$ é a razão capital produto $(K / X)$. Nesse caso, quanto menor for a razão capital produto $(\sigma)$, maior será o impacto negativo da participação do salário no produto $(\lambda)$ sobre o emprego.

Essas equações descrevem a dinâmica cíclica de curto prazo. No longo prazo, a solução do modelo é uma família de ciclos fechados, com o seguinte ponto central $\left(w^{*}\right)$ e $\left(\lambda^{*}\right)$ :

$$
\begin{aligned}
& w^{*}=1-\sigma(x-n) \\
& \lambda^{*}=\left(x+\eta_{1}\right) / \eta_{2}
\end{aligned}
$$

Ambas as equações (3) e (4) são semelhantes às de Lotka e Volterra. Esse sistema representa as características centrais das economias capitalistas para Goodwin. O modelo mostra uma interação entre duas classes que são parcialmente hostis e parcialmente dependentes. Os ciclos de Goodwin seriam, assim, uma expressão de um modus vivendi da relação entre os capitalistas e trabalhadores. ${ }^{3}$

\section{Modelo de Goodwin com envelhecimento populacio- nal}

A riqueza do modelo de Goodwin também é observada em sua aplicação na discussão sobre o impacto das mudanças demográficas no crescimento econômico, como construído por Rada (2012) e que é a base para a análise empírica feita neste estudo. Em seu artigo, Rada (2012) combina um crescimento e uma acumulação cíclica do modelo de Goodwin (1967) com a mudança tecnológica induzida e uma curva salarial ampliada para introduzir os efeitos de um imposto de seguridade social. Através dessa modificação é possível explorar como as mudanças na distribuição secundária de renda, causadas pela crescente taxa de dependência, podem afetar as

3 Orphanides e Solow (1990) também interpretam o modelo de Goodwin como uma interação presa e predador, mas entre trabalhadores empregados e desempregados. 
relações de produção nas economias capitalistas. Seu trabalho desenvolve alguns modelos baseados em dois aspectos. No primeiro, pressupõem-se duas políticas fiscais: na primeira, o imposto de seguridade social é fixo. Nesse caso, o aumento no número de aposentados não provoca aumento no imposto, apenas o ajuste nos benefícios. Na segunda política, o imposto é definido endogenamente. Com isso, o benefício é considerado fixo e modificações no número de beneficiários altera o nível do imposto previdenciário. No segundo aspecto, dois regimes de progresso técnico são considerados: um progresso técnico exógeno e outro induzido.

Rada (2012) estende o modelo de crescimento de Goodwin introduzindo os aposentados e suas aposentadorias no problema da distribuição de renda. De modo geral, a forma como é feita a distribuição primária da renda nacional oferece uma maneira de introduzir a luta de classes e a distribuição de renda na análise da dinâmica econômica a partir do conflito entre salários e lucros.

A renda nacional $(X)$ pode, assim, ser definida da seguinte maneira:

$$
X=W+\Pi
$$

na qual $W$ é igual ao total dos salários na economia e $\Pi$ seria $(r K-\tau K)$, em que $(r K)$ representa o lucro total, sendo r a taxa de lucro, subtraído da depreciação do capital $(\tau K)$. A adição da depreciação do capital no modelo segue as contribuições de Atkinson (1969) e Grasselli, Maheshwari (2018). Reescrevendo-a renda nacional $(X)$ como a participação primária da renda, o que é feito dividindo a equação 5 por $X$, torna-se:

$$
1=w+\pi
$$

em que $\omega=W / X$ é a participação dos salários na renda, e $\pi=(r-\tau) K / X$, a participação dos lucros. $O$ mecanismo nessa equação é simples: um aumento na participação nos salários acarreta diminuição da participação nos lucros, e um aumento na participação dos lucros gera redução na participação dos salários. Em outras palavras, capitalistas e trabalhadores disputam uma parcela maior da renda. Rada (2012) expande essa problemática para incluir um terceiro ator, a população aposentada e pensionista, como um importante grupo na determinação da distribuição de renda. O modelo supõe que o financiamento das pensões vem inteiramente da renda dos 
trabalhadores através de um imposto previdenciário $(\rho)$ aplicado sobre os salários. ${ }^{4}$ Assim, a equação 5 passa a ser escrita da seguinte forma:

$$
X=W(1-\rho)+p R+\Pi
$$

onde $p$ é o nível de pensão e $R$ é o número de aposentados. Dividindo a equação 7 por $L$, obtemos:

$$
x=w(1-\rho)+p d+(r-\tau) k
$$

em que $x$ é a produtividade do trabalho, $d$ a taxa de dependência demográfica $(R / L)$ e $k$ é a razão capital-trabalho. Segundo Rada (2012), a direção e a magnitude da luta de classes também dependerão da natureza do imposto previdenciário. Ela assume dois cenários distintos para $\rho$ : no primeiro, é definido por política em um nível $\bar{\rho}$; e no segundo cenário, o imposto é determinado endogenamente. Partindo dessa contabilidade para uma economia que enfrenta a transição demográfica, Rada (2012) constrói o seu modelo em três blocos: primeiro se define uma função de produção; depois é feita a descrição do funcionamento do mercado de trabalho; e, por fim, o processo de mudança técnica e determinação da produtividade dos fatores.

Por simplicidade, em seu modelo, a economia é formada por um bem único e é representada por uma tecnologia Leontief incorporada em uma função de produção:

$$
f(K, L)=\min [x L, y K]
$$

em que $y=X / K$ é a produtividade do capital. Como se trata de uma função Leontief, logo $X=a L=y K$. A quantidade empregada de mão de obra $(L)$ pode ser derivada da seguinte forma:

$$
L=X / a=K(y / a)
$$

Diferenciando em relação ao tempo, ${ }^{5}$ temos:

4 Cabe destacar, que Rada (2012) também argumenta a possibilidade de um imposto sobre os lucros, mas que não será explorado neste trabalho.

5 Tomando o $\log$ aritmo da equação $L=K(y / a)$, temos $\log L=\log K+\log y-\log a$. Derivan- 


$$
\dot{L} / L=\frac{\dot{K}}{K}+\chi-x
$$

A equação 11 demonstra como a taxa de crescimento da quantidade de mão de obra empregada $(\dot{L} / L)$ está relacionada com a acumulação do capital $(\dot{K} / K)$, a taxa de crescimento da produtividade do capital $(\chi)$, e a taxa de crescimento da produtividade do trabalho é $(x)$. Assumindo que os trabalhadores consomem toda a sua renda, a acumulação de capital é conduzida pelos capitalistas, que poupam todo o seu lucro, a acumulação do capital pode ser descrita da seguinte forma:

$$
\frac{\dot{K}}{K}=\pi y-\tau=(1-\omega) y-\tau .
$$

Com essa nova equação, mudanças na renda em direção a uma melhora na participação dos salários (aumentos em $\omega$ ) tem um efeito adverso sobre a taxa de investimento e, consequentemente, sobre a taxa de crescimento da quantidade empregada de mão de obra. Isso pode ser observado inserindo a equação 12 na equação 11 :

$$
\frac{\dot{L}}{L}=(1-\omega) y-\tau+\chi-x
$$

O segundo bloco do modelo expõe como o mercado de trabalho funciona. De modo simplificado, existem três variáveis principais que caracterizam o mercado de trabalho: o salário real, w; a demanda de trabalho, $L$; e a oferta de trabalho, N. A demanda por trabalho é dada pela equação 13, enquanto a oferta de trabalho é exógena e cresce a uma taxa $\dot{N} / N=n$. Assim, a taxa de crescimento do emprego é dada por:

$$
\dot{\lambda} / \lambda=(1-\omega) y-\tau+\chi-x-n
$$

Seguindo Goodwin (1967), a relação dos salários com o mercado de trabalho é baseada em uma curva de Phillips de salário real, mas agora estendida para explicar os efeitos de um imposto de seguridade social $(\rho)$ sobre a dis-

do ambos os lados em relação ao tempo, encontramos: $\frac{d \log L}{d t}=\frac{d \log K}{d t}+\frac{d \log y}{d t}-\frac{d \log a}{d t}$. Isso implica que: $\dot{L} / L=\frac{\dot{K}}{K}+\chi-\gamma$, sendo $\frac{\dot{K}}{K}, \frac{\dot{y}}{y}=\chi$ e $\frac{\dot{a}}{a}=x$ em que $\dot{K}=\pi \frac{Y}{K}-\tau K$. 
tribuição de renda e, portanto, sobre a acumulação de capital e o emprego. ${ }^{6}$ Nesse sentido, um aumento em $\rho$ após um crescimento na participação dos aposentados leva os trabalhadores a repassarem alguns desses custos para as empresas de acordo com $\dot{w} / \mathfrak{w}=\delta_{1} \lambda+\delta_{2} \rho$, em que $\delta_{1}$ e $\delta_{2}$ são parâmetros positivos acima de 0 . Assim, a participação dos salários segue o movimento:

$$
\dot{\omega} / \omega=\delta_{1} \lambda+\delta_{2} \rho-x
$$

Em síntese, as relações centrais do modelo de Rada (2012) são as equações (14) e (15), aplicadas para explorar dinâmicas macroeconômicas que surgem em uma economia com envelhecimento populacional presente nos quatro modelos testados em seu trabalho. No terceiro bloco do modelo, que foca na mudança tecnológica, são explorados dois casos: no primeiro, assume-se uma mudança tecnológica exógena do tipo Harrod neutro $(\chi=0)$ e no segundo o modelo é ampliado para investigar uma economia com mudança tecnológica endógena.

$\mathrm{Na}$ análise desenvolvida por Rada (2012) são adicionados três variáveis ao modelo original de Goodwin: o imposto de seguridade social, $\rho$, a taxa de crescimento da produtividade do capital $\chi$ e a eficiência de capital $y=X / K=\sigma^{-1}$. Além deles, neste artigo foi adicionado ao modelo a depreciação ( $\tau$ ) Atkinson, 1969; Grasselli; Maheshwari, 2018).

Cada modelo tem a sua solução em um ponto fixo não trivial. No presente modelo testado neste trabalho, com mudança técnica exógena e imposto previdenciário exógeno, a dinâmica da economia depende apenas da interação entre o emprego e a participação dos salários. Ao se imporem as condições $\dot{\lambda}=0$ e $\lambda \neq 0$ na equação 15 , e $\dot{\omega}=0, \omega \neq 0$ e $\chi=0$ na equação 14 , a solução do sistema ocorre no ponto fixo não trivial:

$$
\begin{aligned}
& \lambda^{*}=\left[\left(x-\delta_{2} \rho\right) / \delta_{1}\right. \\
& w^{*}=1-((x+n+\tau) / y
\end{aligned}
$$

Em Rada (2012), o resultado das simulações para esse modelo indica que uma seguridade social exógena não altera as características qualitativas

6 A pesquisa sobre os efeitos dos regimes tributários na fixação de salários e no desemprego (Bovenberg, 2003; Holmlund; Kolm, 1995; Koskela; Schob, 1999; Layard, 1982) motiva a introdução do imposto previdenciário na negociação salarial. 
do modelo de Goodwin. Uma tentativa dos trabalhadores em dividir os custos das pensões e aposentadorias com os capitalistas não tem nenhum efeito sobre a distribuição de renda. No entanto, isso gera uma menor taxa de emprego no estado estacionário em torno do qual a economia oscila. Nesse modelo, níveis maiores de desemprego servem para reduzir ou neutralizar o conflito entre aposentados e trabalhadores. O conflito social em uma economia que enfrenta uma transição demográfica pode dificultar os esforços para garantir a sustentabilidade do sistema previdenciário. Nas próximas seções, o modelo de Goodwin expandido por Rada (2012) é testado com os dados dos países da amostra.

\section{Dados e modelo econométrico}

Para facilitar a exposição, esta seção está dividida em três partes. Em um primeiro momento são apresentadas a base de dados e as variáveis utilizadas no modelo. Na seção 4.2 as metodologias econométricas para estimação dos parâmetros do modelo são discutidas. E por fim, na seção 4.3 são demonstrados os testes estatísticos utilizados.

\subsection{Base de dados}

Neste estudo foram utilizados dados anuais ${ }^{7}$ para nove países: Austrália, Canadá, Dinamarca, Finlândia, França, Grécia, Itália, Reino Unido e EUA, no período de 1960 a 2010, a partir do banco de dados macroeconômicos da AMECO e do banco de dados da OCDE. Trata-se de uma base de dados comumente utilizada nas análises e relatórios da Direção Geral de Assuntos Econômicos e Financeiros da Comissão Europeia. Como este artigo busca testar a aderência do modelo de Goodwin (1967) ao incluir um sistema de seguridade social, optou-se por adotar o mesmo período de análise e parte da amostra dos países ${ }^{8}$ utilizados por Grasselli e Maheshwari (2018).

A primeira variável a ser definida é a produção. Para isso, foi utilizado o Produto Interno Bruto (PIB) ao custo dos fatores (PIBcf) que já está descon-

7 Justificável pela ausência de dados de curto prazo, o que impõe certas limitações para a análise, como apontado por Veneziani e Mohun (2006).

8 Diante da unificação da Alemanha, esse país foi excluído da análise. 
tado de impostos e subsídios sobre a produção e as importações. Assim, o produto real $Y$ é igual ao PIB a preços correntes menos os impostos líquidos sobre a produção e importações, deflacionados com base no deflator do PIB. Além disso, para a estimação do modelo de Goodwin, é preciso repartir a renda real total $(Y)$ em salários reais totais e lucros reais totais. Seguindo Klump et al. (2007), o salário real foi calculado utilizando-se as remunerações dos empregados e a renda dos autônomos. ${ }^{9}$

Os lucros reais totais são definidos como a diferença entre o produto $(Y)$ e os salários reais $(W): \Pi=Y-W$. Para definição da produtividade do trabalho e da taxa salarial, primeiramente definimos o emprego total $(L)$ como sendo o total de empregados mais os trabalhadores por conta própria. Já o total da força de trabalho $(N)$ é definido como o emprego total somado ao total de desempregados. Assim, a produtividade (a) é definida como $Y / L$ e a taxa salarial (w) como $W / L$.

A taxa de dependência dos aposentados $\left(d_{e}=R D R\right)$ foi calculada com base no total da população acima de 65 anos $(R)$ em relação ao emprego total $(L)$, representado por $R / L$. Para a taxa de depreciação $(\tau)$ foi utilizada a definição do manual do banco de dados AMECO: razão entre consumo de capital fixo (preços correntes) e o Capital Social Líquido multiplicado pelo Deflator de Preços da Formação Bruta de Capital Fixo. Por fim, as duas variáveis no estado estacionário, a taxa de emprego $(\lambda)$ e a participação salarial $(\omega)$, são, respectivamente, $L / N$ e $W / Y$. Por se tratar de um equilíbrio não trivial, os valores da taxa de emprego e participação dos salários na renda correspondem aos valores médios ao longo de um ciclo de crescimento, conforme demonstrado em Gandolfo (1980, p. 448).

\subsection{Modelo econométrico}

O modelo econométrico desenvolvido é baseado na contribuição de Grasselli e Maheshwari (2018), mas utilizando as modificações para o modelo

9 Cabe destacar que o Deflator do PIB pode não ser o mais adequado para deflacionar o salário real (W). Conforme Glyn (2009), tradicionalmente a parcela salarial é estimada a preços correntes. Contudo, a base de dados da AMECO somente fornece dados que necessitam de ajuste com uso de deflator, que é calculado da seguinte forma:

$$
W=\left(1+\frac{\text { Autônomos }}{\text { Total de Empregados }}\right) \times \frac{\text { Remuneração dos Empregados }}{\text { Deflator do PIB }}
$$


de Goodwin (1967) desenvolvidos por Rada (2012). A estimativa da taxa de emprego de equilíbrio $(\lambda)$ depende da estimação da curva de Philips $\left(\delta_{1}, \delta_{2}\right)$, do crescimento da produtividade $(x)$ e do imposto previdenciário $(\rho)$. Já a estimativa da participação dos salários de equilíbrio $(\omega)$ dependerá das variáveis $(\chi),(x),(y)$ e $(\beta)$. ○ conjunto dessas estimativas $\left(\hat{\tilde{\delta}}_{1}, \hat{\delta_{2}}, \hat{\tilde{\chi}}, \hat{\tilde{\gamma}}\right.$, $\hat{y}_{1}, \check{\rho}$ e $\left.\breve{n}\right)$ pode ser usado para calcular os "preditores de estimativa econométrica" para $\lambda$ e $\omega$. A eficiência de capital $(y)$ e o imposto previdenciário $(\rho)$ foram encontrados utilizando a média calculada para as duas variáveis. O parâmetro para taxa de crescimento da produtividade e taxa de crescimento populacional pode ser estimado usando o logaritmo das variáveis na tendência temporal das equações (18) e (19).

O comportamento para a produtividade do trabalho e a força de trabalho é fornecido pelas equações:

$$
\begin{aligned}
& a(t)=a_{0} e^{x t} \\
& N(t)=N_{0} e^{n t}
\end{aligned}
$$

Cabe destacar que apenas no equilíbrio trivial, a taxa de crescimento do salário real é $x$. Nesse sentido, a taxa de crescimento da produtividade $(x)$ foi estimada usando a regressão de Mínimos Quadrados Ordinários (MQO). Uma função exponencial semelhante também foi empregada para modelar o crescimento da força de trabalho $(n)$.

$$
\begin{aligned}
& \log \left(a_{t}\right)=\log \left(a_{0}\right)+\hat{x} t+e_{t} \\
& \log \left(N_{t}\right)=\log \left(N_{0}\right)+\hat{n} t+e_{t}
\end{aligned}
$$

No sentido de obter as estimativas $\left(\hat{\delta}_{1}\right)$, e $\left(\hat{\delta}_{2}\right)$ para os parâmetros da curva linear de Philips foi adotado neste estudo o teste de limites para a cointegração dentro da modelagem ARDL. Esse modelo foi desenvolvido por Pesaran et al. (2001) e pode ser aplicado independentemente da ordem de integração das variáveis. Um breve resumo do procedimento envolvido é apresentado aqui. Inicialmente é necessário definir um vetor de variáveis $z_{t}$ :

$$
z_{t}=\left(\eta_{t}, \varphi_{t}^{\prime}\right)^{\prime}
$$


em que $\eta_{t}$ é a variável dependente e $\varphi_{t}$ é um vetor de regressores. $\bigcirc$ processo de geração de dados de $z_{t}$ é uma autorregressão do vetor de ordem $p$. Para a análise de cointegração, é essencial que $\Delta \eta_{t}$ seja modelado como um modelo de Correção de Erros Irrestrito (ECM) condicional:

$$
\Delta \eta_{t}=\beta_{0}+\pi_{\eta \eta} \eta_{t-1}+\pi_{\eta \eta^{*} \varphi} \varphi_{t-1}+\sum_{i=1}^{p} \vartheta_{i} \Delta \eta_{t-1}+\sum_{j=0}^{q} \phi_{j}^{\prime} \Delta \varphi_{t-j}+\theta w_{t}+\mu_{t}
$$

Aqui $\pi_{\eta \eta}$ e $\pi_{\eta \eta \cdot \varphi}$ são multiplicadores de longo prazo. $\beta_{0}$ é a derivada e $w_{t}$ é um vector de componentes exógenos e variáveis fictícias. $\bigcirc$ procedimento de teste de limites testa a ausência de qualquer relação de nível entre $\eta_{t}$ e $\varphi_{t}$ através da exclusão das variáveis de níveis defasados $\eta_{t-1}$ and $\varphi_{t-1}$ na equação 23. Segue-se, então, que o nosso teste para a ausência de uma relação de nível condicional entre $\eta_{t}$ e $\varphi_{t}$ tem as seguintes hipóteses nula e alternativa:

$$
\begin{aligned}
& H_{0}: \pi_{\eta \eta}=0, \pi_{\eta \eta \cdot \varphi}=0^{\prime} \\
& H_{1}: \pi_{\eta \eta} \neq 0, \pi_{\eta \eta \cdot \varphi} \neq 0^{\prime} \text { or } \pi_{\eta \eta} \neq 0, \pi_{\eta \eta \cdot \varphi}=0^{\prime} \text { or } \pi_{\eta \eta}=0, \pi_{\eta \eta \cdot \varphi} \neq 0^{\prime}
\end{aligned}
$$

Essas hipóteses podem ser examinadas usando a estatística $\mathrm{F}$ padrão. $\mathrm{O}$ teste $\mathrm{F}$ tem uma distribuição não padrão que depende:

a) se as variáveis incluídas no modelo ARDL são $I(1)$ ou $I(0)$;

b) do número de regressores;

c) se o modelo ARDL contém um intercepto e/ou uma tendência. Neste estudo, começaremos com a estimativa de um ECM:

$$
\Delta z_{t}=\Phi_{1} z_{t-1}+\Phi_{2} \varphi_{t-1}+\sum_{i=1}^{p} \Phi_{3} \Delta z_{t-i}+\sum_{j=0}^{q} \phi_{4} \Delta \varphi_{t-j}+\mu_{t}
$$

em que $(\varphi)$ é o vetor de regressores, imposto de seguridade social e taxa de emprego. A taxa de crescimento do salário é definida como:

$$
z_{t}=\ln \left(w_{t}\right)-\ln \left(w_{t-1}\right)
$$

E o lag p é determinado usando o critério de informação Bayesiano - Bayesian Information Criterion (BIC) - e o critério de informação - Akaike Information Criterion (AIC). Se esse é o caso, então realizamos um "teste F" da hipótese $H_{0}:=\phi_{1}=\phi_{2}=0$ em relação à alternativa de que $H_{0}$ não é 
verdadeira. A distribuição da estatística de teste é não padrão, e os valores críticos foram dados também em Pesaran et al. (2001). Se encontrarmos valores críticos acima do limiar, então estimaremos o "modelo de níveis" de longo prazo e o ECM restrito para encontrar relações de equilíbrio de longo prazo e efeitos dinâmicos de curto prazo.

\subsection{Testes estatísticos realizados}

Para estimar os parâmetros da curva de Phillips expandida com o imposto previdenciário, dados pela equação 15 , o primeiro passo foi verificar se as variáveis consideradas são estacionárias. $\mathrm{Na}$ Tabela 1 são apresentadas as estimativas do teste aumentado de Dickey-Fuller (teste ADF) para as variáveis: crescimento dos salários reais, taxa de emprego, crescimento da produtividade, inflação, crescimento dos salários nominais e o imposto previdenciário. Com exceção da França, o crescimento dos salários reais e o crescimento da produtividade são estacionários, enquanto a taxa de emprego, a inflação, o crescimento dos salários nominais e o imposto de seguridade social não são estacionários.

Visto que o crescimento dos salários reais e a taxa de emprego têm ordem de integração diferente, não é possível usar modelos de série temporal padrão. Portanto, foi usado o procedimento de teste de limites proposto por Pesaran et al. (2001). A metodologia adotada por ele tem vantagem que pode ser aplicada independentemente se os regressores subjacentes forem puramente $I(0)$, puramente $I(1)$ ou mutuamente cointegrados. No entanto, a maioria das séries temporais são $I(1)$. $\bigcirc$ modelo de Correção de Erros Irrestrito eficaz é dado por:

$$
\Delta z_{t}=\Phi_{0}+\Phi_{1} z_{t-1}+\Phi_{2} \rho_{t-1}+\Phi_{3} \lambda_{t-1}+\Phi_{4} \Delta z_{t-1}+\Phi_{5} \Delta \rho_{t-1}+\Phi_{6} \Delta \lambda_{t-1}+\mu_{t}
$$

Os valores para as defasagens máximas foram determinados utilizando um ou mais dos "critérios de informação" - AIC, SC (BIC), HQ. Uma premissa fundamental na metodologia de Pesaran et al. (2001) é de que os erros da equação 28 devem ser independentes em série. Esse requisito também pode influenciar a escolha final das defasagens máximas para as variáveis no modelo. Neste trabalho, o teste de Breusch-Godfrey foi utilizado para testar a existência de correlação serial no modelo. 
Tabela 1 P-Values para o teste Dickey-Fuller aumentado

\begin{tabular}{lrr|r|r|r|r|r}
\hline País & $\begin{array}{r}\text { Crescimento } \\
\text { do salário } \\
\text { real }\end{array}$ & $\begin{array}{r}\text { Taxa de } \\
\text { emprego }\end{array}$ & $\begin{array}{r}\text { Crescimento } \\
\text { da produti- } \\
\text { vidade }\end{array}$ & $\begin{array}{r}\text { Inflação } \\
\text { Crescimento } \\
\text { do salário } \\
\text { nominal }\end{array}$ & $\begin{array}{r}\text { Imposto } \\
\text { previden- } \\
\text { ciário }\end{array}$ \\
\hline Canadá & 0,0051 & 0,2277 & 0,0004 & 0,1406 & 0,1827 & 0,0145 \\
\hline Dinamarca & 0,0001 & 0,3634 & 0,0000 & 0,4382 & 0,2227 & 0,1979 \\
\hline Finlândia & 0,0027 & 0,4349 & 0,0001 & 0,1452 & 0,2911 & 0,2684 \\
\hline França & 0,1203 & 0,5718 & 0,6331 & 0,5249 & 0,6820 & 0,0000 \\
\hline Grécia & 0,0123 & 0,9377 & 0,0020 & 0,4357 & 0,5746 & 0,7528 \\
\hline Itália & 0,0346 & 0,3634 & 0,0196 & 0,6155 & 0,4884 & 0,2490 \\
\hline Inglaterra & 0,0022 & 0,4945 & 0,0000 & 0,2647 & 0,0809 & 0,1936 \\
\hline EUA & 0,0019 & 0,0518 & 0,0002 & 0,4668 & 0,0466 & 0,0273 \\
\hline
\end{tabular}

Fonte: Elaboração própria.

O procedimento de teste de limites testa a ausência de qualquer relação de nível entre $z_{t}, \rho_{t}$ e $\lambda_{t}$ através da exclusão dos níveis defasados das variáveis $z_{t-1}, \rho_{t-1}$ e $\lambda_{t-1}$ na equação 28 . Segue-se, então, que o teste para a ausência de uma relação de nível condicional entre $z_{t}$ e $\lambda_{t}$ tem as seguintes hipóteses nula e alternativa:

$$
\begin{aligned}
& H_{0}: \phi_{1}=\phi_{2}=\phi_{3}=0 \\
& H_{1}: \phi_{1} \neq \phi_{2} \neq \phi_{3} \neq 0
\end{aligned}
$$

Essas hipóteses podem ser examinadas usando a estatística $\mathrm{F}$ padrão. Os testes F para a significância conjunta dos coeficientes nos níveis de um período defasados das variáveis são comparados com os valores críticos de F presentes em Pesaran et al. (2001). A hipótese nula de não cointegração é rejeitada quando o valor da estatística de teste excede o valor dos limites críticos superiores, e aceita se a estatística $\mathrm{F}$ for menor que o valor dos limites inferiores. Se o teste cai entre os limites, o resultado é inconclusivo.

Nos resultados da estatística $\mathrm{F}^{10}$ para as restrições de articulação $\left(\phi_{1}=\phi_{2}\right.$ $\left.=\phi_{3}=0\right)$, temos em $(k+2)$ três variáveis (crescimento da taxa de salário, taxa de emprego e taxa de previdência social). Então, nas tabelas de teste 10 O resultado para o F-Stat foi: Canadá 6,27; Dinamarca 7,894; França 4,276; Grécia 3,426; Itália 4,549; Inglaterra 5,245 e EUA 2,039. 
de limites de valores críticos em Pesaran et al. (2001), temos $k=1$. Os valores críticos para um modelo com intercepto restrito e nenhuma tendência nos níveis de $1 \%, 5 \%$ e $10 \%$ correspondentes aos valores de $I(0)$ e $I(1)$ são [4,13 e 5,00], [3,10 e 3,87] e [2,63 e 3,35], respectivamente. A estatística F está acima do valor crítico $I(1)$ no nível de significância $1 \%$ para quatro países (Canadá, Dinamarca, Finlândia e GBR). No nível de significância, $5 \%$ França e Itália também estão acima do valor crítico I(1). Na Grécia, a estatística F cai entre os limites, consequentemente, o teste é inconclusivo. Nos EUA, a estatística F calculada cai abaixo do limite inferior; portanto, concluímos que nenhuma cointegração é possível. Como o valor de nossa estatística $\mathrm{F}$ excede o limite superior no nível de significância de $5 \%$, podemos concluir que há evidências de uma relação de longo prazo entre as duas séries temporais (nesse nível de significância ou maior) para todos os países, exceto para a Grécia e os EUA. Em seguida, apresentamos o teste de Ljung Box para verificar a ausência de correlação serial no erro da equação 21. Isso é importante para garantir que o modelo seja bem especificado. Na Tabela 2 observa-se que os valores p são maiores que $10 \%$ para todos os países até o intervalo 5, não implicando correlação serial.

Tabela 2 P-values para correlação serial nos erros ECM irrestrito

\begin{tabular}{llllllll}
\hline País & Lag 1 & Lag 2 & Lag 3 & Lag 4 & Lag 5 \\
\hline Canadá & 0,986 & 0,625 & 0,786 & 0,868 & 0,936 \\
\hline Dinamarca & 0,907 & 0,932 & 0,986 & 0,961 & 0,881 \\
\hline Finlândia & 0,882 & 0,452 & 0,660 & 0,808 & 0,766 \\
\hline França & 0,345 & 0,635 & 0,323 & 0,297 & 0,386 \\
\hline Grécia & 1,000 & 0,997 & 0,980 & 0,995 & 0,988 \\
\hline Itália & 0,678 & 0,876 & 0,942 & 0,977 & 0,976 \\
\hline Inglaterra & 0,941 & 0,880 & 0,841 & 0,513 & 0,624 \\
\hline EUA & 0,953 & 0,934 & 0,901 & 0,952 & 0,688 \\
\hline
\end{tabular}

Fonte: Elaboração própria.

Para calcular os valores de equilíbrio dos parâmetros estimados um último teste a ser realizado é o da hipótese nula de que os coeficientes da regressão estimada são constantes ao longo do tempo. Nesse sentido, os resultados da soma acumulada dos resíduos recursivos (CUSUM test) das equações 26 e 27 em todos os países, à exceção da França, possuem flutuações dentro do intervalo de confiança de $95 \%$. O que nos faz aceitar a 
hipótese nula de que os parâmetros são constantes nessas duas equações durante todo o período.

\section{Resultados encontrados}

Com base no modelo de Rada (2012) e nos dados e modelo econométrico apresentados anteriormente, essa seção do trabalho apresenta os principais resultados encontrados. Para facilitar a apresentação, os resultados foram divididos em duas partes: na primeira é feita uma análise preliminar dos dados e na segunda são apresentados os resultados das estimações econométricas.

\subsection{Análise preliminar dos dados}

Nesta subseção são discutidas as trajetórias das variáveis taxa de emprego $(\lambda)$ e participação dos salários $(\omega)$ para os seguintes países: Austrália, Canadá, Dinamarca, Finlândia, França, Grécia, Itália, Reino Unido e Estados Unidos (EUA), no período de análise que vai de 1960 a 2010. Foram utilizadas informações a partir do banco de dados da AMECO e da OCDE. A existência de padrões consistentes nos dados de emprego e participação nos salários fornece evidências importantes para a avaliação do modelo de Goodwin (Zipperer; Skott, 2011). A média e o desvio padrão das variáveis de participação dos salários e taxa de emprego para o período entre 1960 e 2010 são mostradas na Tabela 3.

A atual trajetória das duas variáveis no período pós-Segunda Guerra é apresentada na Figura 1. Pode-se perceber que as evidências encontradas emprestam certo apoio qualitativo ao modelo de Goodwin para um conjunto de países. Mesmo não apresentando curvas estáveis, um período de elevado emprego é seguido por elevação na participação dos salários na renda, que logo após é substituída por uma queda no nível de emprego e uma redução na participação dos salários, algo previsto nos ciclos de Goodwin. Contudo, chamam a atenção alguns comportamentos específicos.

O primeiro deles são algumas evidências de que os países vivenciaram ciclos de execução mais curtos, que aparentemente giram em torno da tendência de longo prazo. Em função desse comportamento existem relações 
econômicas que geram ciclos sistematicamente, mas que não o fazem historicamente de maneira igual porque podem correr mudanças econômicas e sociais que alteram os parâmetros do modelo (Veneziani; Mohun, 2006). Perceber essas questões não invalida o mecanismo identificado por Goodwin, mas é fundamental para uma interpretação mais cautelosa dos resultados econométricos, visto que uma das suposições assumidas é de que o modelo descreve movimentos de longo prazo.

Tabela 3 Sumário das estatísticas - (1960-2010)

\begin{tabular}{lrr|r|r}
\hline \multirow{2}{*}{ País } & & Taxa de emprego & \multicolumn{2}{r}{ Participação dos salários } \\
\cline { 2 - 5 } & Média & Desvio & Média & Desvio \\
\hline Canadá & $93,19 \%$ & $(2,00 \%)$ & $65,57 \%$ & $(2,65 \%)$ \\
\hline Dinamarca & $95,81 \%$ & $(2,38 \%)$ & $66,17 \%$ & $(2,40 \%)$ \\
\hline Finlândia & $94,56 \%$ & $(3,85 \%)$ & $66,32 \%$ & $(4,74 \%)$ \\
\hline França & $94,46 \%$ & $(3,01 \%)$ & $67,92 \%$ & $(3,45 \%)$ \\
\hline Grécia & $95,36 \%$ & $(2,36 \%)$ & $47,15 \%$ & $(3,19 \%)$ \\
\hline Itália & $93,91 \%$ & $(1,76 \%)$ & $60,29 \%$ & $(3,13 \%)$ \\
\hline Inglaterra & $94,94 \%$ & $(2,78 \%)$ & $64,98 \%$ & $(3,64 \%)$ \\
\hline EUA & $94,46 \%$ & $(1,45 \%)$ & $65,21 \%$ & $(1,71 \%)$ \\
\hline
\end{tabular}

Fonte: Elaboração própria, com base nos dados da OCDE.

Um segundo comportamento que pode ser observado é que na série histórica analisada todos os países apresentaram nível de taxa de emprego menor ao final do período (2010) do que no início (1960). Já em relação à participação dos salários na renda, à exceção da Grécia e Dinamarca, todos os demais países apresentaram piora ao final do período quando comparado com o início. Apesar de não confirmar o movimento previsto na análise original de Goodwin, que pressupunha ciclos fechados, essas evidências são um indício de que há tendência de aumento da massa de trabalhadores que vai sendo colocada à margem do sistema econômico (ampliação do Exército Industrial de Reserva), o que é mais próximo de uma tendência apontada pelo próprio Marx, e que está diretamente relacionada com o atual período de globalização neoliberal, como destacado por Cooney (2008). 
Figura 1 Taxa de emprego e participação dos salários (1960-2010)

(a) Canadá

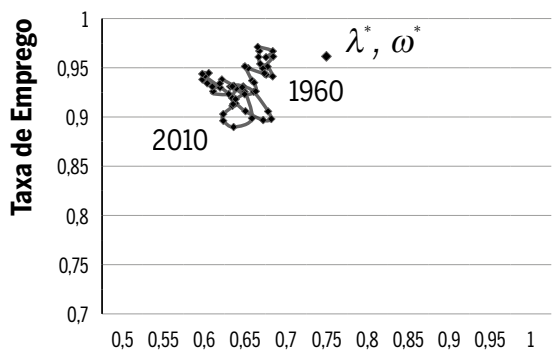

(c) Finlândia

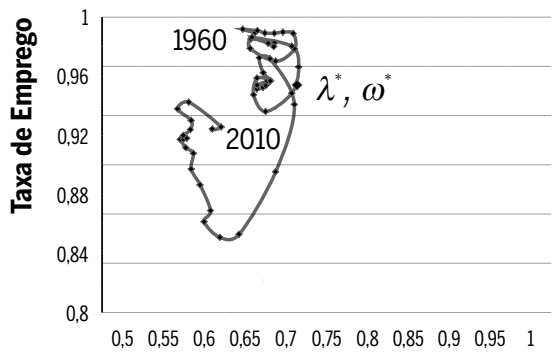

(e) Grécia

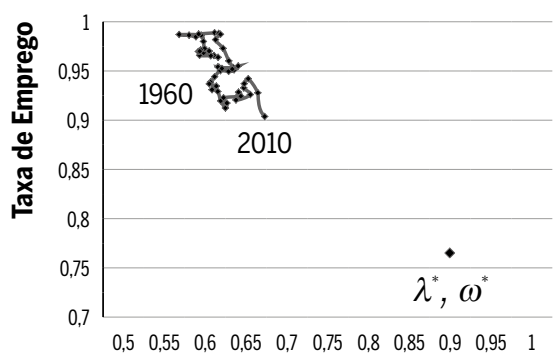

(g) Inglaterra

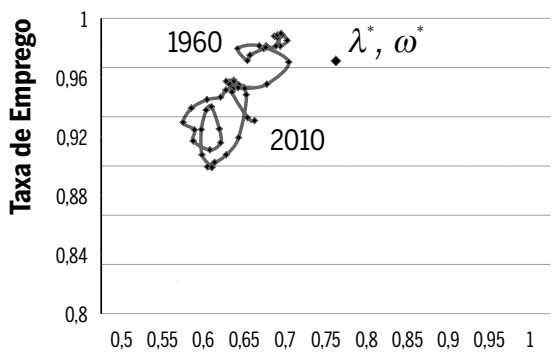

Fonte: Elaboração própria. (b) Dinamarca

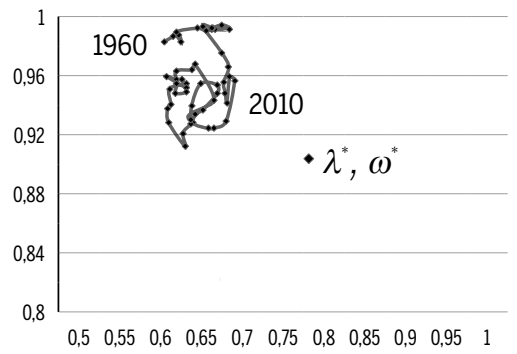

(d) França

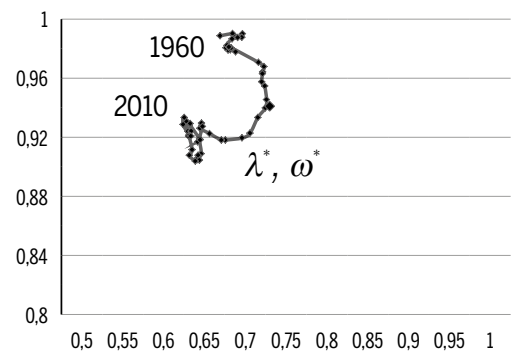

(f) Itália

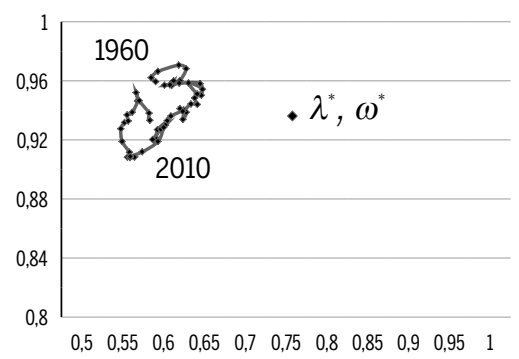

(h) USA

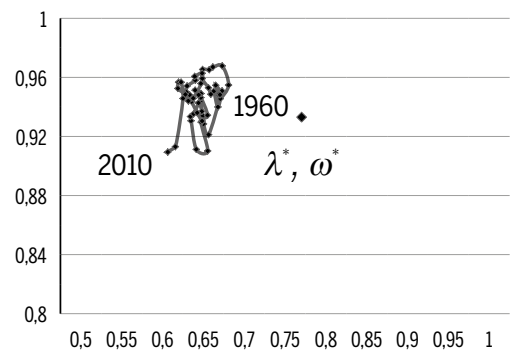

Participação nos Salários 


\subsection{Resultados das estimações}

Os parâmetros de crescimento da força de trabalho (equação 20) e crescimento da produtividade (equação 21) foram feitos pelo método de MOO. Os resultados das estimativas são mostrados na Tabela 4 e na Tabela 5.

Tabela 4 Estimação do crescimento da força de trabalho

\begin{tabular}{lrrrrrrr|r|r}
\hline País & Canadá & Dinamarca & Finlândia & França & Grécia & Itália & Inglaterra & EUA \\
\hline $\operatorname{InN}_{0}$ & 8,998 & 7,867 & 7,927 & 10,081 & 8,501 & 10,133 & 10,15 & 11,27 \\
\hline $\boldsymbol{\beta}$ & 0,020 & 0,004 & 0,0008 & 0,005 & 0,005 & 0,004 & 0,006 & 0,016 \\
\hline $\mathbf{R}$ & 0,973 & 0,853 & 0,143 & 0,977 & 0,616 & 0,707 & 0,944 & 0,974 \\
\hline adjR & 0,972 & 0,850 & 0,126 & 0,976 & 0,608 & 0,701 & 0,943 & 0,973 \\
\hline Fstat & $1,791,43$ & 286,19 & 8,21 & 2083,41 & 78,66 & 118,25 & 830,39 & 1851,14 \\
\hline LBQstat & 48,63 & 40,23 & 43,29 & 38,69 & 43,11 & 38,68 & 45,12 & 46,20 \\
\hline JBStat & 4,82 & 0,76 & 3,64 & 2,06 & 2,63 & 20,02 & 6,47 & 2,48 \\
\hline
\end{tabular}

Fonte: Elaboração própria, com base nas estimações.

Tabela 5 Estimação do crescimento da produtividade

\begin{tabular}{lrrrrrrrr|r|r}
\hline País & Canadá & Dinamarca & Finlândia & França & Grécia & Itália & Inglaterra & EUA \\
\hline Ina 0 & 3,809 & 5,382 & 2,686 & 3,043 & 2,413 & 3,031 & 2,885 & 3,747 \\
\hline$\alpha$ & 0,012 & 0,019 & 0,032 & 0,024 & 0,026 & 0,022 & 0,019 & 0,015 \\
\hline $\mathbf{R}$ & 0,925 & 0,979 & 0,971 & 0,911 & 0,776 & 0,814 & 0,981 & 0,978 \\
\hline adjR & 0,923 & 0,978 & 0,970 & 0,909 & 0,771 & 0,810 & 0,981 & 0,978 \\
\hline Fstat & 607,80 & 2260,15 & 1670,04 & 501,12 & 169,87 & 214,65 & 2635,05 & 2213,37 \\
\hline LBQstat & 41,92 & 32,08 & 39,09 & 43,54 & 43,17 & 42,77 & 32,38 & 39 \\
\hline JBStat & 1,09 & 3,23 & 3,97 & 4,76 & 1,57 & 5,49 & 0,44 & 0,53 \\
\hline
\end{tabular}

Fonte: Elaboração própria, com base nas estimações.

As estimações dos parâmetros do crescimento da produtividade e da força de trabalho vão na mesma direção da tendência apresentada por esses países nas últimas décadas, mas cabem aqui algumas considerações especiais. No que diz respeito ao crescimento da força de trabalho, é possível identificar duas tendências ao longo da recente história dos países da OCDE (OECD, 2012). Uma que vai até meados de 1980 e outra a partir desse período. Na primeira, os países apresentam uma taxa de crescimento da força de trabalho mais elevada (com valores ao redor de $1 \%$ a.a.). Já na segunda, há uma queda no crescimento da força de trabalho, sendo que alguns paí- 
ses apresentam valores negativos. Essa tendência de baixo crescimento da força de trabalho reflete a redução do índice de natalidade, não compensada pelos processos migratórios, que aliada ao aumento na expectativa de vida cria o cenário demográfico que pressiona as contas previdenciárias na maior parte desses países.

Em relação à produtividade do trabalho, os valores também se aproximam das tendências apresentadas por esses países durante o período (OECD, 2013, 2016). Cabe destacar que, diferentemente das nações subdesenvolvidas, em especial da América Latina, esses países não sofrem de heterogeneidade estrutural em suas economias, o que torna os níveis de produtividade dos diferentes setores relativamente mais próximos entre si do que em países como o Brasil, por exemplo.

Tabela 6 Estimativas de longo prazo para a curva de Phillips

\begin{tabular}{|c|c|c|c|c|}
\hline País & Variável & $\delta_{2}$ & $\delta_{1}$ & AdjR2 \\
\hline \multirow{2}{*}{ Canadá } & Coeff & $-0,780$ & 0,047 & \multirow{2}{*}{0,4306} \\
\hline & $\mathrm{p}$-Value & 0,0098 & 0,0002 & \\
\hline \multirow{2}{*}{ Dinamarca } & Coeff & $-1,528$ & 0,015 & \multirow{2}{*}{0,6094} \\
\hline & $\mathrm{p}$-Value & 0,0036 & 0,0057 & \\
\hline \multirow{2}{*}{ Finlândia } & Coeff & $-0,353$ & 0,068 & \multirow{2}{*}{0,5208} \\
\hline & $\mathrm{p}$-Value & 0,0004 & 0,0000 & \\
\hline \multirow{2}{*}{ França } & Coeff & $-0,649$ & 0,133 & \multirow{2}{*}{0,2349} \\
\hline & $\mathrm{p}$-Value & 0,0058 & 0,0036 & \\
\hline \multirow{2}{*}{ Grécia } & Coeff & $-1,053$ & 0,128 & \multirow{2}{*}{0,2351} \\
\hline & $\mathrm{p}$-Value & 0,0519 & 0,0168 & \\
\hline \multirow{2}{*}{ Itália } & Coeff & $-1,637$ & 0,223 & \multirow{2}{*}{0,2779} \\
\hline & $\mathrm{p}$-Value & 0,0013 & 0,0009 & \\
\hline \multirow{2}{*}{ Inglaterra } & Coeff & $-0,498$ & 0,049 & \multirow{2}{*}{0,3613} \\
\hline & $\mathrm{p}$-Value & 0,05 & 0,0002 & \\
\hline \multirow{2}{*}{ EUA } & Coeff & $-0,232$ & 0,030 & \multirow{2}{*}{0,2294} \\
\hline & p-Value & 0,0009 & 0,004 & \\
\hline
\end{tabular}

Fonte: Elaboração própria, com base nas estimações.

Como já foi identificado que as variáveis são cointegradas, pode-se "extrair" efeitos de longo prazo do ECM irrestrito. Olhando para a equação 22 , os coeficientes de longo prazo para $\delta_{2}$ e $\delta_{1}$ são $-\left(\phi_{2} / \phi_{1}\right)$ e $-\left(\phi_{3} / \phi_{1}\right)$, respectivamente. Na Tabela 6 apresentamos as estimativas de longo prazo da curva linear de Phillips (equação 28). 


$$
z_{t}=\hat{\delta}_{2} \rho_{t}+\hat{\delta}_{1} \lambda_{t}
$$

Finalmente, as estimativas de equilíbrio do modelo de Goodwin expandido por Rada (2012) podem ser mostradas na Tabela 7. Os parâmetros de produtividade e taxa de crescimento populacional (a) e (n) são retirados das Tabelas 4 e 5 , respectivamente. $\bigcirc$ imposto previdenciário $(\rho)$ é a média dos pagamentos obrigatórios históricos pagos às administrações públicas. Da mesma forma, o índice de eficiência de capital ( $y)$ é a média histórica da razão entre o produto real e o estoque de capital. As variáveis $\left(\delta_{1}\right)$ e $\left(\delta_{2}\right)$ são os parâmetros da curva linear de Phillips estimadas na Tabela 6. Assim, as variáveis $\lambda_{G}$ e $\omega_{G}$ são as estimativas de equilíbrio final do modelo de Goodwin estendido derivado das Equações 16 e 17.

Tabela 7 Sumário dos valores dos parâmetros e o equilíbrio estimado

\begin{tabular}{lrr|r|r|r|r|r|r|r|r}
\hline País & $\boldsymbol{\alpha}$ & $\boldsymbol{\beta}$ & $\boldsymbol{y}$ & $\boldsymbol{\rho}$ & $\boldsymbol{\delta}_{\mathbf{2}}$ & $\boldsymbol{\delta}_{\mathbf{1}}$ & $\boldsymbol{\tau}$ & $\boldsymbol{\lambda}_{\boldsymbol{G}}$ & $\boldsymbol{\omega}_{\boldsymbol{G}}$ \\
\hline Canadá & $1,3 \%$ & $2,0 \%$ & 0,398 & $4,10 \%$ & $-0,780$ & 0,047 & $6,13 \%$ & $96,17 \%$ & $76,3 \%$ \\
\hline Dinamarca & $1,9 \%$ & $0,4 \%$ & 0,341 & $0,33 \%$ & 1,528 & 0,015 & $4,81 \%$ & $90,43 \%$ & $79,1 \%$ \\
\hline Finlândia & $3,2 \%$ & $0,1 \%$ & 0,281 & $9,31 \%$ & $-0,353$ & 0,068 & $4,29 \%$ & $95,42 \%$ & $73,1 \%$ \\
\hline França & $2,4 \%$ & $0,5 \%$ & 0,302 & $15,64 \%$ & $-0,649$ & 0,133 & $4,89 \%$ & $94,12 \%$ & $74,2 \%$ \\
\hline Grécia & $2,6 \%$ & $0,5 \%$ & 0,273 & $8,29 \%$ & $-1,053$ & 0,128 & $3,32 \%$ & $88,65 \%$ & $76,5 \%$ \\
\hline Itália & $2,2 \%$ & $0,4 \%$ & 0,319 & $11,43 \%$ & $-1,637$ & 0,223 & $4,71 \%$ & $93,61 \%$ & $77,1 \%$ \\
\hline Inglaterra & $1,9 \%$ & $0,6 \%$ & 0,322 & $5,68 \%$ & $-0,498$ & 0,049 & $4,72 \%$ & $97,12 \%$ & $77,6 \%$ \\
\hline EUA & $1,5 \%$ & $1,6 \%$ & 0,371 & $5,75 \%$ & $-0,232$ & 0,030 & $4,96 \%$ & $93,31 \%$ & $78,3 \%$ \\
\hline
\end{tabular}

Fonte: Elaboração própria, com base nas estimações.

Os resultados das estimativas da participação dos salários e do emprego $\left(\lambda_{G}\right.$ e $\left.\omega_{G}\right)$ foram plotados na Figura 1. Em linha com os resultados identificados por Grasselli e Maheshwari (2017; 2018), há um melhor ajuste das estimações do que em Harvie (2000). As estimativas para a taxa de emprego de equilíbrio $\left(\lambda_{G}\right)$ estão dentro do limite dos valores observados durante o tempo de estudo. A diferença do equilíbrio estimado da média observada para a taxa de emprego está abaixo de 1 ponto percentual para três países: Finlândia, França e Grécia. Inglaterra e EUA estavam dentro do desvio padrão. A Dinamarca e a Grécia mostraram as maiores diferenças entre o parâmetro e a média histórica, 5,38\% e 6,71\%, respectivamente. $O$ presente trabalho mostrou diferenças entre os valores estimados e a média histórica bem próximas de Grasselli e Maheshwari (2018), que também 
apresentam um melhor ajuste dos estimadores em relação às médias históricas do que em Harvie (2000), em que nenhuma estimativa estava dentro do desvio padrão. Em relação às estimativas da participação do salário de equilíbrio $\left(\omega_{G}\right)$, nenhum país teve valor de equilíbrio dentro do ciclo observado de longo prazo, resultado diferente de Grasselli e Maheshwari (2018), que como nas estimativas para taxa de emprego também tiveram um bom ajuste em relação à média histórica. Contudo, cabe destacar que o erro diminuiu drasticamente, se comparado a Harvie (2000). Com exceção da Grécia, que mostrou uma diferença de 29 pontos percentuais, todos os países ficaram entre 2-16 pontos percentuais, em comparação com 20-100 pontos percentuais relatados por Harvie (2000).

Além das mudanças metodológicas, a melhora nos resultados do modelo testado neste trabalho indica que o processo de envelhecimento populacional também pode influenciar o conflito em torno da distribuição de renda e, consequentemente, os resultados do modelo de Goodwin. Segundo o modelo mais simples desenvolvido por Rada (2012) e testado nesse trabalho - que considera o imposto previdenciário exógeno (relativamente fixo) e o nível de benefícios ajustáveis -, a tentativa dos trabalhadores de repartirem os custos das pensões e aposentadorias com os capitalistas não tem repercussões sobre a participação nos lucros, mas afeta o nível de emprego no estado estacionário, reduzindo-o. Esse resultado sugere que em contextos de envelhecimento populacional o conflito social dificulta os esforços de sustentar economicamente a população de inativos.

\section{Considerações finais}

O presente artigo teve como objetivo explorar os impactos que o envelhecimento populacional provoca no ciclo econômico via sistema de seguridade social, tomando como referência o modelo de Goodwin expandido por Rada (2012) e aplicado para algumas economias desenvolvidas. Com essa motivação, o artigo buscou construir uma contribuição à literatura que trata de aspectos empíricos da área de pesquisa que se estabeleceu a partir do trabalho seminal de Goodwin (1967). Para isso, além da apresentação formal do modelo, também foram discutidos os fundamentos teóricos e metodológicos que subsidiam o modelo de Goodwin, bem como algumas evidências qualitativas para um conjunto de países da OCDE. 
A originalidade do artigo é encontrada na aplicação da metodologia econométrica proposta em Harvie (2000), e posteriormente retificada por Grasselli e Maheshwari (2018), para analisar o ganho de aderência empírica do modelo de Goodwin (1967) quando se incorpora a esse modelo um sistema de seguridade social caracterizado por uma alíquota de imposto previdenciária homogênea e exogenamente determinada e níveis de pensão individuais que se ajustam recorrentemente, em um dado número de aposentados. A principal conclusão aponta que mesmo a versão mais simples do modelo de Goodwin expandida por Rada (2012) apresentou um bom ajustamento empírico, tendo apresentado uma melhora da aderência empírica quando comparada à versão original de Goodwin analisada por Harvie (2000). Contudo ela ainda pode ser ampliada com alguns ajustes propostos por Grasselli e Maheshwari (2018), como a adição da variável taxa de acumulação de capital no modelo.

Além dessas evidências, a discussão aqui desenvolvida também aponta para outras questões importantes. Apesar das diversas limitações dentro dessa abordagem, a capacidade do modelo em traduzir um fenômeno central na dinâmica econômica, que é o conflito entre as classes em torno da distribuição de renda, em uma linguagem matemática simplificada é um dos seus pontos fortes. A existência de uma crescente literatura heterodoxa utilizando-o reforça a importância do arcabouço marxista em entender as sociedades capitalistas contemporâneas e a sua dinâmica, o que justifica a contínua e frutífera pesquisa nesse campo do pensamento econômico, bem como a sua relação com outras teorias no campo heterodoxo.

\section{Referências}

ARAUJO, R. A.; DÁVILA-FERNANDEZ, M. J.; MOREIRA, H. N. Some new insights of the empirics of Goodwin's growth-cycle model. Structural Change and Economics Dynamics, v. 51, p. 42-54, 2019.

ATKINSON, A. B. The timescale of economic models: How long is the long run? The Review of Economic Studies, Oxford, v. 36, n. 2, p. 137-152, 1969.

BATISDAS, D.; FABRE, A.; ISAAC, F. Minskyan classical growth cycles: Stability analysis of a stock-flow consistent macrodynamic model. Mathematics and Financial Economics, v. 13, p. 359-391, 2019.

BOVENBERG, A. L. Tax policy and labor market performance. Tilburg: Tilburg University, Sept. 2003. (Working Paper, 1.035). 
COONEY, P. Uma avaliação empírica da lei geral da acumulação capitalista no período atual de globalização neoliberal. Revista de Economia, v. 34, n. especial, p. 51-76, 2008.

DÁVILA-FERNÁNDEZ, M. J.; LIBÂNIO, G. A. Goodwin cycles and the BopC growth paradigm: A macrodynamic model of growth and fluctuations. EconomiA, v. 17, p. 324-339, 2016.

FLASCHEL, P. The Goodwin distributive cycle after fifteen years of new observations. In topics in classical micro- and macroeconomics. Springer Berlin Heidelberg, Berlin, v. 3, n. 17, p. 465-480, 2009.

GANDOLFO, G. Economic Dynamics: Methods and Models. New York: North-Holland, 1980. $592 \mathrm{p}$.

GLYN, A. Functional distribution and inequality. In: NOLAN, B. SALVERDA, W. SMEEDING, T. (Ed.). The Oxford Handbook of Economic Inequality. Oxford: Oxford University Press, 2009. p. 101-126.

GOLDSTEIN, J. P. Predator-Prey model estimate of the cyclical profit squeeze. Metroeconomica, v. 50, n. 2, p. 139-217, 1999.

GOODWIN, R. M. A growth cycle. In: FEINSTEIN, C. H. (Ed.). Socialism, capitalism and economic growth. London: Cambridge University, 1967, p. 78-91.

GRASSELLI, M.; MAHESHWARI, A. A comment on 'testing Goodwin: Growth cycles in ten OECD countries'. Cambridge Journal of Economics, v. 41, n. 6, p. 1.761-7.166, 2017.

GRASSELLI, M. R.; MAHESHWARI, A. Econometric estimation of Goodwin growth models. Metroeconomica. v. 69, n. 3, p. 619-643, 2018.

HARVIE, D. Testing Goodwin: Growth cycles in ten OECD countries. Cambridge Journal of Economics, Cambridge, v. 24, n. 3, p. 349-476, 2000.

HOLMLUND, B.; KOLM, A. Progressive taxation, wage setting, and unemployment: Theory and Swedish evidence. Swedish Economic Police Review, Stockholm, v. 2, p. 423-460, 1995.

KLUMP, R.; MCADAM, P.; WILMAN, A. Factor substitution and factor augmenting technical progress in the U.S. Review of Economics and Statistics, Washington, v. 89, p. 183-192, 2007.

KOSKELA, E.; SCHOB, R. Does the composition of wage and payrol taxes matter under Nash bargaining? Applied Economic Letters, Amsterdan, v. 64, p. 343-349, Apr. 1999.

LAYARD, R. Is Income policy the answer to unemployment? Economica, Washington, v. 49, p. 219-239, 1982.

MARX, K. Capital. Harmondsworth: Penguin, 1976. v. 1.

MOLINA, M. G.; MEDINA, E. H. Are there Goodwin employment-distribution cycles? International empirical evidence. Cuadernos de Economia, v. 29, n. 53, p. 1-29, 2010.

ORGANISATION FOR ECONOMIC COOPERATION AND DEVELOPMENT (OECD). OECD Employment Outlook. Paris: OECD, 2012.

ORGANISATION FOR ECONOMIC COOPERATION AND DEVELOPMENT (OECD). OECD Compendium of Productivity Indicators 2016. Paris: OECD, 2016.

ORGANISATION FOR ECONOMIC COOPERATION AND DEVELOPMENT (OECD). Pensions at a Glance 2013: OECD and 20 Indicators. Paris: OECD, 2013. 
ORPHANIDES, A.; SOLOW, R. M. Money, inflation and growth. In: FRIEDMAN, B. M.; HAHN, F. H. (Ed.). Handbook of Monetary Economics. New York: Elsevier, 1990, p. 223-261.

PESARAN, M. H.; SHIN, Y; SMITH, R. J. Bounds testing approaches to the analysis of level relationships. Journal of Applied Econometrics, Piscataway, v. 16, p. 289-326, 2001.

PICHARDO, G. Notas para las sesiones. Porto Alegre, maio de 2009. 104 p. (Curso: Mudança Estrutural e Crescimento Econômico).

RADA, C. Social security tax and endogenous technical change in an economy with aging population. Metroeconomica, Oxford, v. 63, n. 4, p. 727-756, 2012.

RAMMELT, C. F. The dynamics of financial instability: Simplifying Keen's Goodwin-Minsky model. System Dinamic Review, v. 35, n. 2, p. 140-159, 2019.

SÁNCHEZ, D; ESTEVÃO, E.; CARVALHO, L.; MIEBACH, A.; CECCONELLO, M.. Goodwin economic cycle via p-fuzzy system. Journal of Intelligent \& Fuzzy Systems, v. 38, p. 4.079-4.090, 2020.

SHAIKH, A. Capitalism, Competition, Conflict, Crises. New York: Oxford University Press. 2016.

SPORTELLI, M. A Kolmogoroff generalizad predator-prey model of Goodwin's growth cycle. Zeitschrift fur Nationalokonomie, Berlin, v. 61, n. 1, p. 35-64. 1995.

TARASSOW, A. The empirical relevance of Goodwin's business cycle model for US economy. MPRA Paper 21012. Munich: University Library of Munich, 2010.

TAYLOR, L.; FOLEY, D.; REZAI, A. Demand drives growth all the way: Goodwin, Kaldor, Pasinetti and the Steady State. Cambridge Journal of Economics, v. 1, p. 1-20, 2018.

VENEZIANI, R. Structural Stability and Goodwin's a Growth Cycle: A Survey. London: London Scholl of Economics and Political Sciences, 2001.

VENEZIANI, R.; MOHUN, S. Structural stability in Goodwin's growth cycle. Structural Change and Economic Dynamics, Amsterdan, v. 17, n. 2, p. 437-451, 2006.

ZIPPERER, B; SKOTT, P. Cyclical patterns of employment, utilization, and profitability. Journal of Post Keynesian Economics, Oxford, v. 34, n. 1, p. 25-57, 2011.

\section{Sobre os autores}

Daniel NogueiraSilva - daniel.nogueira@unifesspa.edu.br

Instituto de Estudos em Desenvolvimento Agrário e Regional, Universidade Federal do Sul e Sudeste do Pará, Marabá, PA, Brasil.

ORCID: https://orcid.org/0000-0002-8379-4672.

HenriqueMorrone-hmorrone@hotmail.com

Departamento de Economia, Universidade Federal do Rio Grande do Sul, Porto Alegre, Rio Grande do Sul, Brasil. ORCID: https://orcid.org/0000-0001-9579-8489.

Aos pareceristas da revista Nova Economia, que deram importantes contribuições para a versão final deste artigo.

\section{Sobre o artigo}

Recebido em 11 de março de 2020. Aprovado em 11 de outubro de 2020. 